\title{
Inhibition of platelet aggregation by carbon monoxide-releasing molecules (CO-RMs): comparison with NO donors
}

\author{
Stefan Chlopicki • Magdalena Lomnicka • \\ Andrzej Fedorowicz • Elżbieta Grochal • \\ Karol Kramkowski • Andrzej Mogielnicki • \\ Włodzimierz Buczko • Roberto Motterlini
}

Received: 2 October 2011 / Accepted: 21 January 2012/Published online: 25 February 2012

(C) The Author(s) 2012. This article is published with open access at Springerlink.com

\begin{abstract}
Carbon monoxide (CO) and CO-releasing molecules (CO-RMs) inhibit platelet aggregation in vitro. Herein, we compare the anti-platelet action of CORM-3, which releases CO rapidly $\left(t_{1 / 2} 1 \mathrm{~min}\right)$, and CORM-A1, which slowly releases $\mathrm{CO}\left(\mathrm{t}_{1 / 2}=21 \mathrm{~min}\right)$. The anti-platelet effects of $\mathrm{NO}$ donors with various kinetics of $\mathrm{NO}$ release were studied for comparison. The effects of CO-RMs and NO donors were analyzed in washed human platelets (WP), platelets rich plasma (PRP), or whole blood (WB) using aggregometry technique. CORM-3 and CORM-A1 inhibited platelet aggregation in human PRP, $\mathrm{WP}$, or WB, in a concentration-dependent manner. In all three preparations, CORM-A1 was more potent than CORM-3. Inhibition of platelets aggregation by CORM-A1 was not significantly affected by a guanylate cyclase inhibitor (ODQ) and a phosphodiesterase-5 inhibitor, sildenafil. In contrast, inhibition of platelet aggregation by NO donors was more potent with a fast $\mathrm{NO}$ releaser (DEA-NO, $t_{1 / 2}=2 \mathrm{~min}$ ) than slow
\end{abstract}

S. Chlopicki $(\bowtie) \cdot$ M. Lomnicka $\cdot$ E. Grochal

Department of Experimental Pharmacology, Chair

of Pharmacology, Jagiellonian University Medical College,

Krakow, Poland

e-mail: s.chlopicki@jmrc.org.pl

S. Chlopicki · A. Fedorowicz $\cdot$ E. Grochal

Jagiellonian Centre for Experimental Therapeutics (JCET),

Jagiellonian University,

Krakow, Poland

K. Kramkowski $\cdot$ A. Mogielnicki $\cdot$ W. Buczko

Pharmacodynamics, Medical University in Bialystok,

Bialystok, Poland

R. Motterlini

INSERM U955, Faculté de Médecine, Université Paris Est,

Creteil, France
NO releasers such as PAPA-NO $\left(t_{1 / 2}=15 \mathrm{~min}\right)$ or other slow NO donors. Predictably, the anti-platelet effect of DEA-NO and other NO donors was reversed by ODQ while potentiated by sildenafil. In contrast to $\mathrm{NO}$ donors which inhibit platelets proportionally to the kinetics of $\mathrm{NO}$ released via activation of soluble guanylate cyclase (sGC), the slow CO-releaser CORM-A1 is a superior anti-platelet agent as compared to CORM-3 which releases $\mathrm{CO}$ instantly. The anti-platelet action of CO-RMs does not involve sGC activation. Importantly, CORM-A1 or its derivatives representing the class of slow $\mathrm{CO}$ releasers display promising pharmacological profile as anti-platelet agents.

Keywords CO-releasing molecule (CO-RMs) · Platelet aggregation $\cdot \mathrm{NO}$ donors $\cdot$ Human platelets
Abbreviations
CORM-3 Tricarbonylchloro(glycinato)ruthenium(II)
CORM-A1 Sodium boranocarbonate
DEA-NO Diethylammonium (Z)-1-( $N, N$-diethylamino) diazen-1ium-1,2-diolate
DETA-NO (Z)-1-[N-(2-aminoethyl)- $N$-(2-ammonioethyl) amino]diazen-1-ium-1,2-diolate
DPTA-NO (Z)-1-[N-(3-aminopropyl)- $N$-(3- ammoniopropyl)amino]diazen-1-ium-1, 2-diolate
iCORM-A1 CORM-A1 deactivated by nitrogen bubbling in acidified solution
L-NAME $\quad N$-nitro-L-arginine methyl ester
ODQ 1H-[1,2,4]Oxadiazolo[4,3-a]quinoxalin-1-one
PAPA-NO (Z)-1-[N-(3-aminopropyl)- $N$-( $n$-propyl)amino] diazen-1-ium-1,2-diolate
SNAP $\quad S$-nitroso- $N$-acetyl penicillamine 


\section{Introduction}

Endogenous carbon monoxide (CO) formation in mammals is catalyzed by a family of heme oxygenase enzymes (HO), an inducible isoform (HO-1) and a constitutive protein (HO-2), that decompose heme into $\mathrm{CO}$, ferrous iron, and biliverdin, the latter being converted to bilirubin by biliverdin reductase (Maines and Kappas 1977; Tenhunen et al. 1969). CO is recognized as a signalling molecule within the cardiovascular system being involved in the regulation of vascular tone as well as exerting anti-inflammatory, anti-apoptotic, antiatherogenic, anti-proliferative, and cytoprotective activities (Motterlini and Otterbein 2010). The biological effects of $\mathrm{CO}$, mainly manifested through vascular smooth muscle relaxation and mitigation of inflammatory processes, involve stimulation of soluble guanylate cyclase (sGC) and cyclic guanosine monophosphate (cGMP) production (Furchgott and Jothianandan 1991; Utz and Ullrich 1991), activation of calcium-dependent potassium channels (Wang and Wu 1997), stimulation of p38 mitogen-activated protein kinase (MAPK) (Otterbein et al. 2000; Otterbein et al. 2003), and direct binding to the heme moiety of structural and functional proteins such as iNOS (Foresti and Motterlini 1999; Sawle et al. 2005).

Carbon monoxide-releasing molecules (CO-RMs), of which the chemical and biochemical features have been thoroughly characterized by Motterlini and co-workers (Johnson et al. 2003; Motterlini 2007; Motterlini et al. 2003), liberate CO in biological systems providing a useful research tool for exploring the mechanism by which $\mathrm{CO}$ exerts its pharmacological activities (Motterlini et al. 2002). Two of these compounds, tricarbonylchloro(glycinato)ruthenium(II) (CORM-3) and sodium boranocarbonate (CORM-A1), have unique features as they are fully water-soluble and have been shown to simulate the bioactivities of gaseous $\mathrm{CO}$ including vessel relaxation (Clark et al. 2003; Foresti et al. 2003), protection against organ ischemia-reperfusion injury (Clark et al. 2003; Foresti et al. 2004; Guo et al. 2004), prevention of organ rejection following transplantation (Clark et al. 2003), and inhibition of the inflammatory response (Sawle et al. 2005).

Importantly, CORM-3 is a metal carbonyl complex that rapidly liberates $\mathrm{CO}$ in physiological buffers (half-life $<1 \mathrm{~min}$ ) (Clark et al. 2003), while CORM-A1 that does not possess a transition metal but a carboxylic moiety liberates $\mathrm{CO}$ at a much slower rate (half-life $21 \mathrm{~min}$ ) under physiological conditions (Motterlini et al. 2005). Recently, we have shown that CORM-3 inhibits platelet aggregation in vitro through a mechanism that is independent of soluble guanylate cyclase activation (Chlopicki et al. 2006). The aim of the present study was to use the two mostly characterized water-soluble $\mathrm{CO}-\mathrm{RMs}$ to evaluate how the different rates of $\mathrm{CO}$ release affect platelet aggregation in vitro and finally compare their anti-platelet profile with the one exerted by NO donors possessing various kinetics of NO release.

\section{Materials and methods}

Detection of CO release by assessing carbonmonoxy myoglobin formation

The release of CO from CORM-3 and CORM-A1 was assessed spectrophotometrically by measuring the kinetics of conversion of deoxymyoglobin (deoxy-Mb) to carbonmonoxy myoglobin (MbCO) as described previously (Motterlini et al. 2003). Myoglobin solutions $(50 \mu \mathrm{mol} / 1$ final concentration) were prepared freshly by dissolving the protein in $0.1 \mathrm{~mol} / 1$ phosphate buffer (pH 7.4). Sodium dithionite $(0.1 \%)$ was added to convert myoglobin to deoxy-Mb prior to each experiment. $\mathrm{CO}$ released from CORM-3 and CORM-A1 (final concentrations: 10, 30, $40 \mu \mathrm{M}$ ) was quantified by adding aliquots of stock solutions $(10 \mu \mathrm{l})$ of the compounds (in pure distilled water) directly to the deoxy-Mb. The kinetics of $\mathrm{MbCO}$ formation was quantified by measuring the change in absorbance at $541 \mathrm{~nm}$ (background wavelength $558 \mathrm{~nm}$ ) for deoxy-Mb at $37^{\circ} \mathrm{C}$ using a spectrophotometer equipped with kinetic software package. The rates of $\mathrm{MbCO}$ formation were calculated and expressed as change in $\mathrm{MbCO}$ concentration $(\mu \mathrm{M})$.

\section{Measurement of $\mathrm{CO}$ release from CORMs using a $\mathrm{CO}$ electrode}

Blood specimens $(20 \mathrm{ml})$ were collected on the day of experiment from healthy volunteers. Specimens were added in 5-ml volumes to TEKLAB ${ }^{\mathrm{TM}}$ blood collection tubes containing $8.75 \mathrm{mg}$ of the anti-coagulant tripotassium EDTA (1.75 per $\mathrm{ml}$ of blood). These specimens were then gently mixed by inversion for $10 \mathrm{~min}$ to guarantee complete solubilizing of the tripotassium EDTA.

Each blood sample was centrifuged for $15 \mathrm{~min}$ at $100 \times \mathrm{g}$ at $4^{\circ} \mathrm{C}$ resulting in the three following layers: the inferior layer composed of red cells, the intermediate layer composed of white cells, and the superior layer made up of plasma. The plasma layer was examined for red cells. If red cells were present, the sample was re-centrifuged for an additional $5 \mathrm{~min}$. The plasma layer was removed and retained as platelet rich plasma (PRP). The remaining blood specimen was then centrifuged at $1,500 \times g$ for $20 \mathrm{~min}$ to collect the platelet poor plasma layer.

The release of CO from CORM-A1 and CORM-3 in buffer or PRP was detected using a prototype electrode purchased from World Precision Instrument (WPI; Stevenage, Herts, UK) and used as previously described (Motterlini et al. 2005). This CO electrode is a membrane-covered amperometric sensor which has been designed on a basic operating principle 
similar to the nitric oxide (NO) sensor. The $\mathrm{CO}$ sensor can be connected to the WPI ISO-NO Mark II meter for detection of the current signals providing that the poise potential is set to a different value $(900 \mathrm{mV}$ for $\mathrm{CO}$ as opposed to $860 \mathrm{mV}$ for $\mathrm{NO}$ ). The electrode was immersed into either $1 \mathrm{ml}$ of buffer solution or $1 \mathrm{ml}$ of PRP and was equilibrated at $37^{\circ} \mathrm{C}$ for $30 \mathrm{~min}$ prior to addition of CO-RMs. Once equilibrated, the electrode was zeroed, and CO-RM ( 0.3 or $3 \mathrm{mM}$ ) was added. Readings were taken until the chart reached the maximal $\mathrm{CO}$ release, and data are expressed as an average of three independent experiments.

Measurement of carbonmonoxy hemoglobin in the whole blood upon exposure to CO-RMs

CORM-3 or CORM-A1 was added to the whole blood, and after 2 or $10 \mathrm{~min}$ of incubation, carbonmonoxy hemoglobin $(\mathrm{COHb})$ content in the blood was analyzed using automatic blood gases analyzer (NovoMedica). After 2 and 10 min of incubation at $36^{\circ} \mathrm{C}$, blood samples $(100 \mu \mathrm{l})$ were placed in a CO-oximeter (Ciba-Corning 270, Siemens), where the blood was hemolyzed with UV and $\mathrm{COHb}$ level was determined. Before first and after last run of blood with CO-RM, three runs with control blood were performed. The zero point was set before first run and after automatically, every $30 \mathrm{~min}$ with Wash/Zero solution (Bayer). The calibration was performed once in a month with CO-OX Slope solution (Bayer), according to producer instruction.

\section{Platelet aggregation assay}

\section{Isolation of human platelets}

Venous blood was obtained from human volunteers at the University Hospital Blood Bank Centre. Volunteer donors had not taken any medicines for the preceding 2 weeks. Blood was collected into vials containing sodium citrate $(3.2 \%, 9: 1 \mathrm{v} / \mathrm{v}$ or $3.8 \%, 10: 1 v / v$ ) as an anti-coagulant agent.

For the platelet aggregation in full blood, venous blood samples were diluted with $0.9 \% \mathrm{NaCl}(1: 1 v / v)$. To obtain PRP, blood was centrifuged at $250 \times g$ for $20 \mathrm{~min}$. Washed platelets (WP) were obtained from PRP which were washed twice in $\mathrm{PGI}_{2}$-containing phosphate buffered saline (PBS) according to the method of Radomski et al. (1988) and finally suspended $\left(2 \times 10^{8}\right.$ platelets $\left./ \mathrm{ml}\right)$ in $\mathrm{Ca}^{2+}$-free PBS containing $0.1 \%$ albumin and $0.1 \%$ glucose. Contamination of neutrophils in WP was less than $1 / 10^{8}$ (Chlopicki et al. 2004).

\section{Platelet aggregation assay in humans}

Aggregation of blood platelets in the whole blood was assessed using Chronolog aggregometer (Chrono-log Corp., USA) by measurements of electrical impedance according to the method described by Cardinal and Flower (1980), while in PRP and WP, it was assessed by measurements of optic transmittance according to the method described by Born (1967).

Whole blood was equilibrated for $13 \mathrm{~min}$, and then, CORM-A1, CORM-3, or VEH $(0.9 \% \mathrm{NaCl})$ were added. Collagen was added after further 2 min of incubation. The dose of collagen was chosen to cause $50 \%$ of full aggregation $\left(\mathrm{EC}_{50}\right)$. The stirrer speed was set at $800 \mathrm{rpm}$. Changes in impedance were registered 6 min after stimulation with collagen. The maximal extension of the aggregation curve at sixth minute was expressed as a percentage of control value.

PRP $(500 \mu \mathrm{l})$ was equilibrated for $2 \mathrm{~min}$ at $37^{\circ} \mathrm{C}$ with continuous stirring at $1,100 \mathrm{rev} / \mathrm{min}$ and then stimulated with collagen to cause aggregation. At the beginning of each experiment, concentrations of collagen that induced sub-maximum aggregation response were determined. These were in the range of $0.5-1.5 \mu \mathrm{g} / \mathrm{ml}$. CORM-3, CORM-A1, $\mathrm{RuCl}_{3}$, inactive form of CORM-A1, DEA-NO, PAPA-NO, DETA-NO, DPTA-NO, and SNAP were added usually 2 min before stimulation of platelets with collagen unless longer incubation was indicated. In some experiments, ODQ $(10 \mu \mathrm{M})$ or sildenafil $(100 \mathrm{nM})$ was added $1 \mathrm{~min}$ prior to the addition of CORMs or NO donors.

WP $(500 \mu \mathrm{l})$ was equilibrated for $2 \mathrm{~min}$ at $37^{\circ} \mathrm{C}$ with continuous stirring at $1,100 \mathrm{rev} / \mathrm{min}$ in PBS containing $\mathrm{CaCl}_{2}$ and $\mathrm{MgCl}_{2}$ both at a concentration of $1 \mathrm{mM}$ and then stimulated with thrombin to cause aggregation. At the beginning of each experiment, concentrations of thrombin that induced sub-maximum aggregation response were determined. These were in the range 15-20 mU/ml. CORM-3, CORM-A1, DEANO, PAPA-NO, DETA-NO, and DPTA-NO were added 2 min before stimulation of platelets unless longer incubation time was indicated. In some experiments, ODQ or sildenafil was added 1 min prior to the addition of CORMs or NO donors.

Reagents and drugs

Collagen was obtained from Chrono-Par (USA), thrombin from Biomed (Poland), DEA-NO (half-life $\sim 2 \mathrm{~min}$ at $37^{\circ} \mathrm{C}$ and $\mathrm{pH}=$ 7.4), PAPA-NO (half-life $\sim 15 \mathrm{~min}$ ), DPTA-NO (half-life $\sim 3 \mathrm{~h}$ ), DETA-NO (half-life $\sim 20 \mathrm{~h}$ ), and ODQ were purchased from Cayman (USA). $\mathrm{Ru}(\mathrm{CO})_{3} \mathrm{Cl}$ (glycinate) (CORM-3) and sodium boranocarbonate $(\mathrm{Na}[\mathrm{H} 3 \mathrm{BCO} 2 \mathrm{H}]$ or $\mathrm{CORM}-\mathrm{A} 1)$ were synthesized as previously described (Clark et al. 2003; Foresti et al. 2003) (Motterlini et al. 2005). The inactive form of CORM-A1 which does not release CO (iCORM-A1) was prepared as described previously (Motterlini et al. 2005). Ruthenium chloride $\left(\mathrm{RuCl}_{3}\right)$ was used as a negative control for CORM-3.

Statistical analysis

All calculations were performed with GraphPad Prism software. Values of $(\mathrm{lg}) \mathrm{IC}_{50}$ were calculated by sigmoidal dose-response 
curve fitting (variable slope, and fixed bottom and top values to 0 and 100 , respectively). Least squares method was used unless the residuals did not follow normal distribution; in this case, robust fit was used. However, this last method did not report confidence intervals for estimated parameters. Comparison of active form of CORMs with inactive counterparts and influence of ODQ or sildenafil on action of CORMs or NO donors were assessed by Mann-Whitney test or with KruskalWallis test followed by Dunn's multiple comparisons. Results of whole blood aggregation were compared by unpaired $t$ test.

\section{Results}

Characteristics of CO release from CORM-3 and CORM-A1

As detected by the myoglobin assay (Fig. 1a), CORM-3 released $\mathrm{CO}$ almost instantly in buffer (half-life $<1 \mathrm{~min}$ at $37^{\circ} \mathrm{C}, \mathrm{pH}$ 7.4), while CORM-A1 released $\mathrm{CO}$ at much slower rate (half-life $\approx 21 \mathrm{~min}$ at $37^{\circ} \mathrm{C}, \mathrm{pH}$ 7.4). Although the $\mathrm{CO}$ electrode detected the release of $\mathrm{CO}$ from CORM-A1 in PRP, which occurred in a slow fashion as previously described in buffer, this device was not sensitive enough to detect measurable amounts of CO from CORM-3 (Fig. 1b). Scavenging of $\mathrm{CO}$ by plasma constituents interacting with CORM-3 could represent one possible reason for the failure of the $\mathrm{CO}$ electrode to detect any CO in PRP. Alternatively, CORM-3 releases CO effectively only in the presence of an avid acceptor, as in the case of reduced myoglobin. In fact, as exemplified in Fig. 1c, there was a striking difference in $\mathrm{CO}$ detection also from CORM-A1 between PRP and buffer solutions showing much less CO being measured in PRP (Fig. 1c). When CO-RMs were incubated in the whole blood for few minutes, we found that $\mathrm{COHb}$ increased from $1.5 \pm 0.17 \%$ (control) to $2.6 \pm 0.15 \%$ with $1 \mathrm{mM}$ CORM-3, while $300 \mu \mathrm{M}$ CORM-A1 did not significantly changed this parameter $(1.76 \pm 0.17 \%)$.

Effects of CORM-3 and CORM-A1 on human platelet aggregation in PRP, WP, and whole blood

As shown in Fig. 2a, when added to human PRP, CORM-A1 or CORM-3 inhibited platelet aggregation induced by collagen in a concentration-dependent manner. However, CORM-A1 was effective in the micromolar concentrations range, while CORM-3 was effective only at millimolar concentrations $\left(\mathrm{IC}_{50}=160.3 \mu \mathrm{M}\right.$ and $3170 \mu \mathrm{M}, 95 \% \mathrm{CI}$ from 2900 to $3464 \mu \mathrm{M}$, for CORM-A1 and CORM-3, respectively). Similarly, the inhibitory effect of CORM-A1 on thrombin-induced platelet aggregation in WP exceeded that of CORM-3 (Fig. 2b). Even though approximately ten times lower concentrations of both CORM-3 and CORM-A1 were a

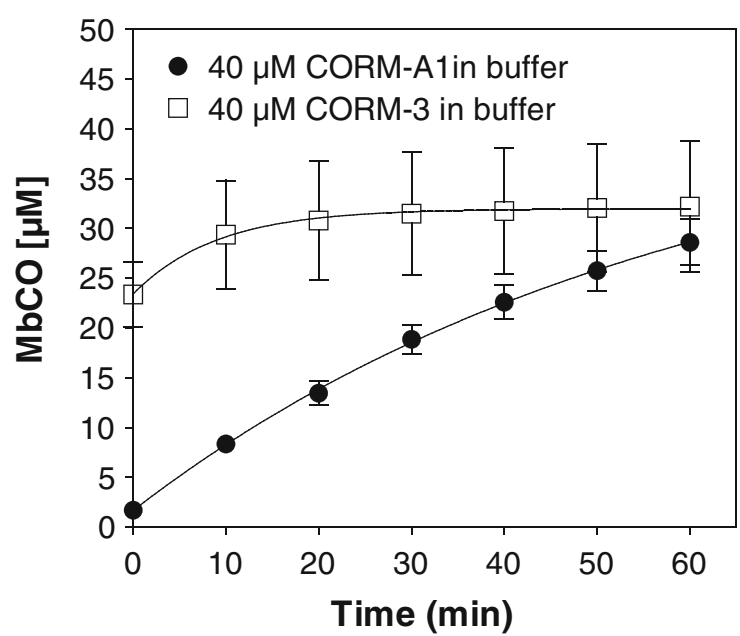

b

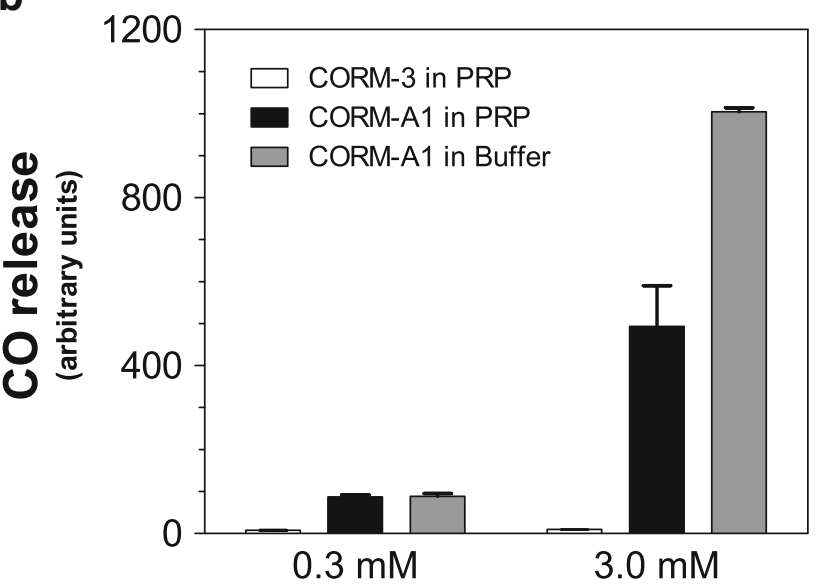

C

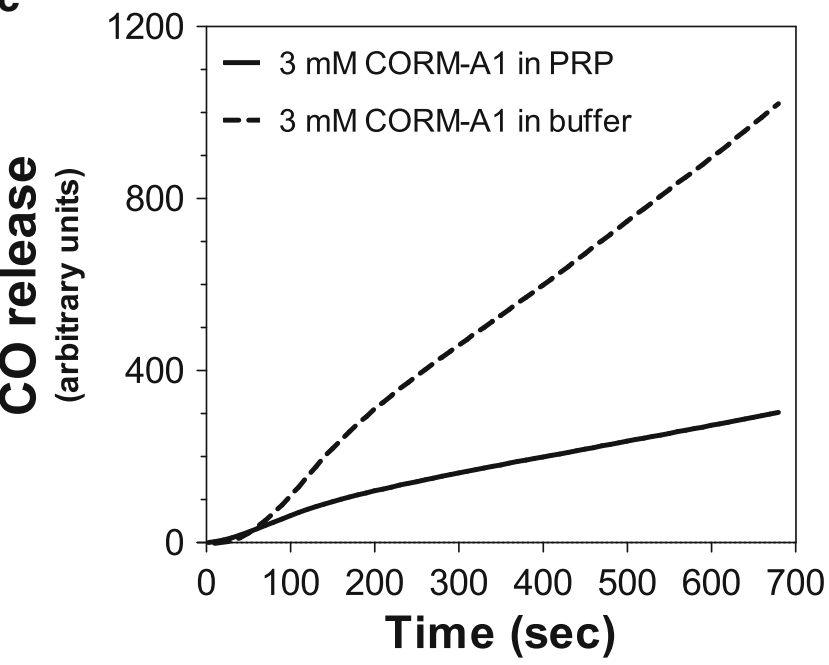

Fig. 1 Time course of MbCO formation from CORM-3 and CORM$\mathrm{A} 1$ in buffer (a) and comparison of CO-releasing capacity from CORM-3 in PRP and CORM-A1 in PRP and Krebs buffer (b). The time course of releasing CO by CORM-A1 in buffer and PRP (c) 
Fig. 2 Concentration-dependent anti-aggregatory effects of CORM-3 and CORM-A1 in human PRP (a) $(n=4-52)$, human WP (b) $(n=4-39)$, and human whole blood in vitro (c) $(n=3-9)$

needed to inhibit washed human platelets as compared to PRP, CORM-3 was more than a tenfold weaker anti-platelet agent than CORM-A1 in WP preparation $\left(\mathrm{IC}_{50}=13.82 \mu \mathrm{M}\right.$ and $171.9 \mu \mathrm{M}$ for CORM-A1 and CORM-3, respectively, Table 1). Also, the inhibitory effect of CORM-A1 on collagen-induced platelet aggregation in vitro human whole blood exceeded that of CORM-3 (Fig. 2c), though the difference in potency was approximately by twofold $\left(\mathrm{IC}_{50}=\right.$ $467.45 \mu \mathrm{M}$ and $1004.16 \mu \mathrm{M}$ for CORM-A1 and CORM-3, respectively; Table 1). Thus, even though the potency of CORM-A1 and CORM-3 differed in PRP, WP, and the whole blood, CORM-A1 was a more potent anti-platelet agent than CORM-3 in human PRP, WP, as well as in the whole human blood.

For the comparison of the anti-platelet activities of CORM-3 and CORM-A1 in PRP and WP, both compounds were incubated for 2 min prior to the addition of collagen (PRP) or thrombin (WP) (Fig. 2a, b). If the incubation time for CORM-3 was prolonged to 5 or $10 \mathrm{~min}$ prior to the stimulation of platelet aggregation either in PRP (Fig. 3a) or WP (data not shown), the anti-platelet activity of CORM-3 was not at all potentiated. In contrast, if CORM-A1 was pre-incubated for 5 or $10 \mathrm{~min}$ prior to the stimulation of platelet aggregation in PRP (Fig. 3a), the anti-platelet effect of CORM-A1 was more accentuated. Similarly, prolongation of CORM-A1 incubation in WP accentuated its anti-aggregatory effects $(8.167 \pm 1.01 \%$ and $25.17 \pm 1.96 \%$ after 2 and $5 \mathrm{~min}$ of incubation with $30 \mu \mathrm{M}$ of CORM-A1, respectively; $p<0.001, n=5-6$ ). The difference in activity of CORM-A1 but not CORM-3 with the prolonged pre-incubation is fully compatible with the instant release of $\mathrm{CO}$ from CORM-3 (half-life $<1 \mathrm{~min}$ at $37^{\circ} \mathrm{C}, \mathrm{pH} 7.4$ ) and the slower rate of $\mathrm{CO}$ release from CORM-A1 (half-life $\approx 21 \mathrm{~min}$ at $37^{\circ} \mathrm{C}, \mathrm{pH}$ 7.4). The respective negative controls used for CORM-3 ( $\left.\mathrm{RuCl}_{3}\right)$ and CORM-A1 (iCORM-A1) that do not possess $\mathrm{CO}$-dependent activity were inactive as anti-platelet agents (Fig. 3b).

Effects of various NO donors on human platelet aggregation in PRP and WP

The comparison of anti-platelet activity of various NO donors (DEA-NO, PAPA-NO, DETA-NO, and DPTA-NO) in PRP is shown in Fig. 4. Both in PRP and WP, the NO donor with the shortest half-life was a more potent inhibitor of platelet aggregation than $\mathrm{NO}$ donors possessing a slower rate of $\mathrm{NO}$ release. In PRP, DEA-NO, $\left(t_{1 / 2}=2 \mathrm{~min}\right.$ at $37^{\circ} \mathrm{C}$ and $\left.\mathrm{pH}=7.4\right)$, PAPANO $\left(t_{1 / 2}=15 \mathrm{~min}\right)$, DPTA-NO $\left(t_{1 / 2}=3 \mathrm{~h}\right)$, and DETA-NO $\left(t_{1 / 2}=\right.$ $20 \mathrm{~h}$ ) inhibited platelets with an $\mathrm{IC}_{50}$ of $0.24 \mu \mathrm{M}, \mathrm{IC}_{50}=$ $0.48 \mu \mathrm{M}, \mathrm{IC}_{50} 8.33 \mu \mathrm{M}$, and $\mathrm{IC}_{50}=38.9 \mu \mathrm{M}$, respectively.
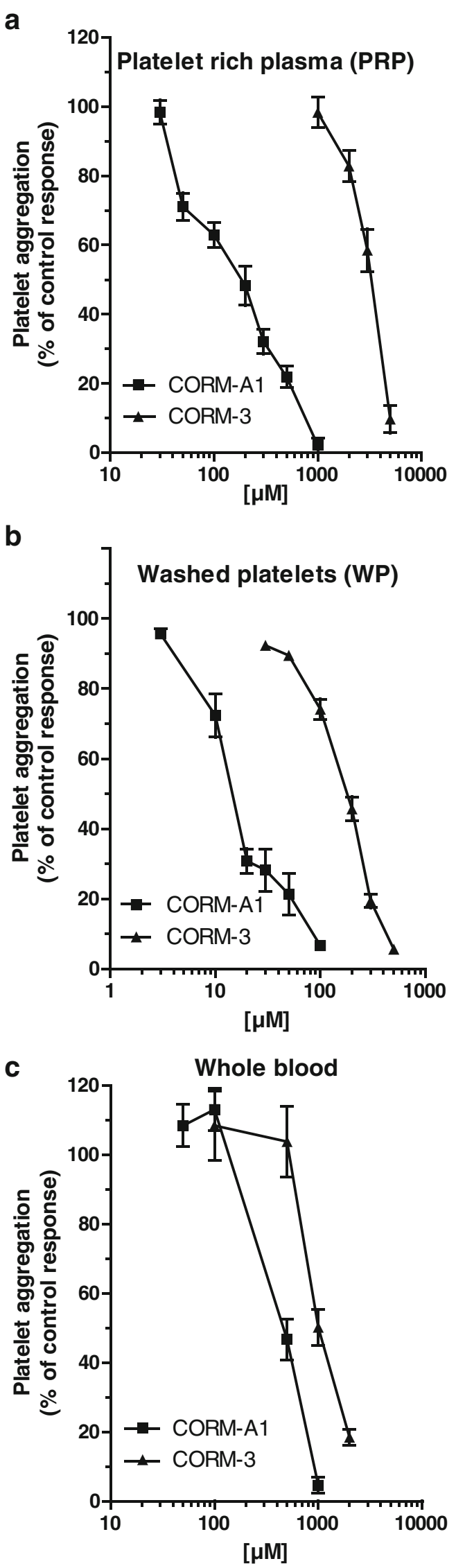
Table $1 \mathrm{IC}_{50}$ values for inhibition of platelets aggregation by CO-RMs and NO donors in whole blood, platelet rich plasma, and washed platelets. All values are interpolated from concentration-dependent curves with the exception of $\mathrm{IC}_{50}$ for DETA-NO in PRP which was extrapolated from the concentration-dependent curve since even at the concentration of $30 \mathrm{mM}$ DETA-NO inhibited platelet aggregation by approximately $40 \%$

\begin{tabular}{|c|c|c|c|c|}
\hline \multirow[b]{2}{*}{ CO-RMs } & \multirow[b]{2}{*}{$t_{1 / 2}$} & \multicolumn{3}{|c|}{ Inhibition of platelet aggregation $\left(\mathrm{IC}_{50}\right)$} \\
\hline & & Whole blood $(\mu \mathrm{M})$ & Platelet rich plasma $(\mu \mathrm{M})$ & Washed platelets $(\mu \mathrm{M})$ \\
\hline CORM-3 & $<1 \mathrm{~min}$ & 1004.2 & 3170.0 & 171.9 \\
\hline CORM-A1 & $21 \mathrm{~min}$ & 467.4 & 160.3 & 13.8 \\
\hline \multicolumn{5}{|l|}{ NO donors } \\
\hline DEA-NO & $2 \min$ & N.D. & 0.24 & 0.022 \\
\hline PAPA-NO & $15 \min$ & N.D. & 0.48 & 0.051 \\
\hline DPTA-NO & $3 \mathrm{~h}$ & N.D. & 8.33 & 0.37 \\
\hline DETA-NO & $20 \mathrm{~h}$ & N.D. & 38.9 & 6.5 \\
\hline
\end{tabular}

N.D. not determined

A similar order of potency for these four NO donors was observed in WP (Table 1).

Differential involvement of soluble guanylate cyclase in the anti-platelet action of CO-RMs and NO donors

As shown in Fig. 5a, an inhibitor of soluble guanylate cyclase activity (ODQ) reversed the inhibition of platelet aggregation induced by DEA-NO, while sildenafil profoundly enhanced the inhibition of platelet aggregation induced by DEA-NO, both in PRP (Fig. 5a) and in WP (data not shown). ODQ and sildenafil displayed similar effects on platelet response to other NO donors (e.g., SNAP; data not shown).

In contrast, in the presence of an inhibitor of soluble guanylate cyclase activity (ODQ), the effect of CORM-A1 was fully preserved either in collagen-stimulated platelets aggregation in PRP (Fig. 5b) or in thrombin-induced platelet aggregation in WP (data not shown). In the presence of a selective inhibitor of phosphodiesterase-5 (sildenafil), the anti-platelet effect of CORM-A1 in PRP (Fig. 5b) and in WP (data not shown) was not potentiated.
Fig. 3 a Influence of incubation time on the antiaggregatory effect of CORM-3 and CORM-A1 in PRP (a) $(n=7-14), 2$ mM CORM-3 and $100 \mu \mathrm{M}$ CORM-A1 were used, respectively). b Lack of the anti-aggregatory effect of inactive forms of CO-RMs in PRP ( $n=5-46)$. One asterisk and three asterisks denote statistical significance vs. standard incubation of $2 \mathrm{~min}$

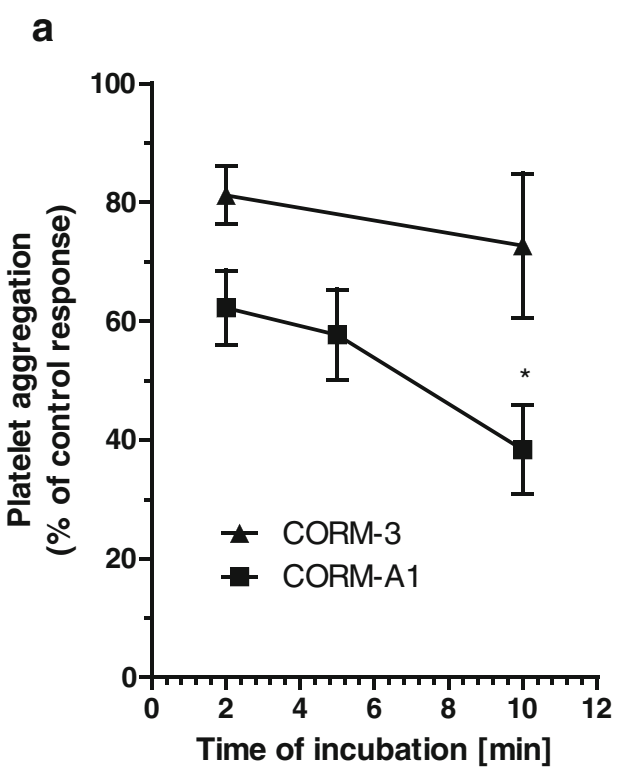

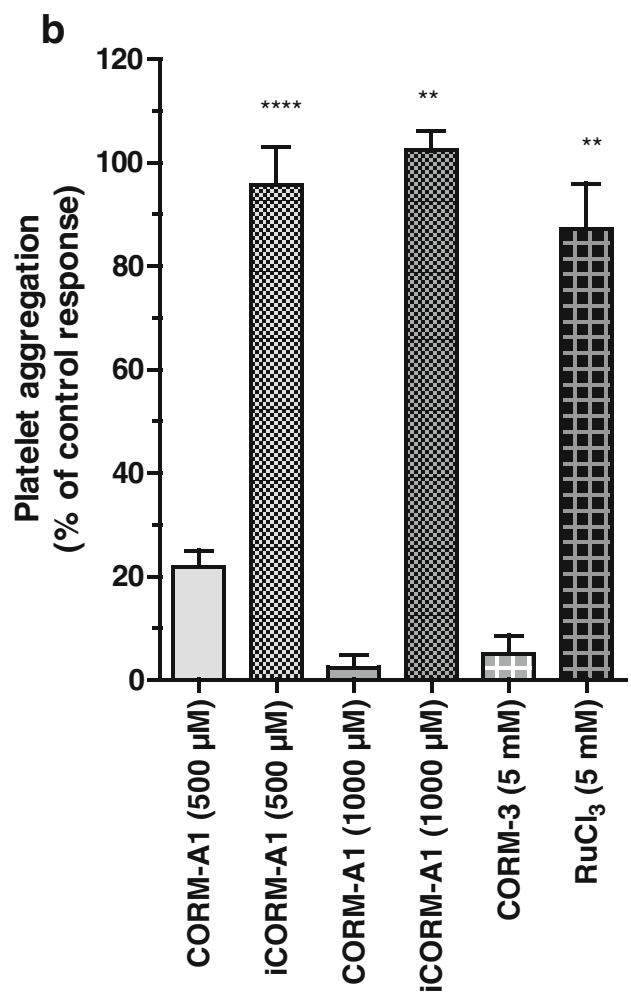




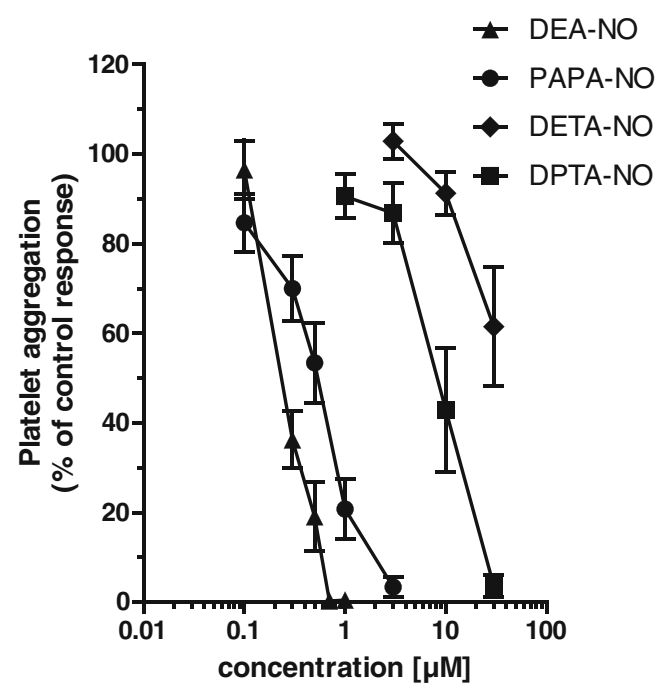

Fig. 4 Concentration-dependent anti-aggregatory effect of NO donors in PRP

\section{Discussion}

In the present work, we evaluated the time- and concentrationdependent anti-platelet effects of two water-soluble COreleasing agents (Motterlini et al. 2002; Motterlini et al.
2003; Motterlini et al 2005). These two compounds are (1) a metal carbonyl complex that rapidly liberates CO (CORM-3) and (2) a novel releaser of $\mathrm{CO}$ that does not contain a transition metal (sodium boranocarbonate, CORM-A1) and generates $\mathrm{CO}$ at a much slower rate under physiological conditions. Using three different in vitro systems for platelet aggregation (PRP, WP, and whole blood), we demonstrated a superior anti-platelet profile of activity for CORM-A1 compared to CORM-3. Indeed, the anti-aggregatory effects of CORM-A1 in PRP and whole blood were achieved in a concentration range that does not significantly affect the formation of $\mathrm{COHb}$. Furthermore, prolongation of pre-incubation of platelets with CORM-A1 but not CORM-3 potentiated the observed effect suggesting that effective anti-aggregatory action by micromolar concentration of CORM-A1 may be achieved through a gradual liberation of $\mathrm{CO}$ over time. Taking into account that CORM-3 elicited prompt and rapid vasodilatory effects in vitro and in vivo whereas CORM-A1 promoted prolonged vasodilation and milder hypotensive effect in vivo (Motterlini et al. 2005), one can speculate that CORM-A1 could afford an anti-aggregatory effect in vivo without a hypotensive effect and possibly without a toxic effect related to increased $\mathrm{COHb}$ formation which might compromise oxygen delivery to tissues. Predictably, exogenous
Fig. 5 Effect of ODQ $(10 \mu \mathrm{M})$ $(n=11-14)$ and sildenafil (100 nM) $(n=7-12)$ on the antiaggregatory action of DEA-NO (a) $(n=3-16)$ and CORM-A1 (b) $(n=7-14)$ in PRP

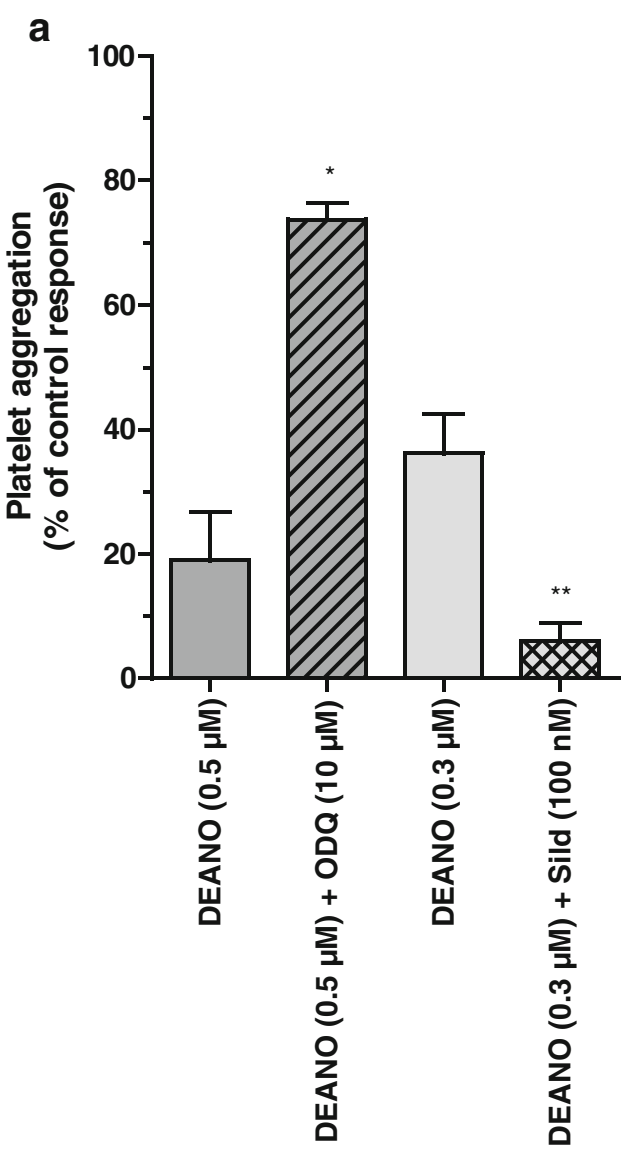


NO affords simultaneously hypotensive and anti-aggregatory effects on platelets, which are both mediated by activation of sGC. In the present work, the pharmacological effects of both CO-RMs on platelets do not appear to involve activation of soluble sGC supporting our previous work on CORM-3 and pointing out that the identification of a specific target for $\mathrm{CO}$ in platelets remains a challenge (Chlopicki et al. 2006).

The biological activities of $\mathrm{CO}$ and $\mathrm{NO}$ are frequently compared. Here, we underscore clear differences on the antiaggregatory properties of CO-RMs and NO donors that could reflect the biological activity of endogenous $\mathrm{NO}$ and $\mathrm{CO}$ on platelet aggregation. Endogenous NO instantly produced by the vascular endothelium can be stimulated by increased blood flow, shear stress, or an agonist, and the rapid kinetics of endogenous NO release has been detected in biological systems (Kalinowski et al. 2003). On the other hand, our body is continuously exposed to small quantities of $\mathrm{CO}$ produced endogenously during the degradation of heme by heme oxygenase enzymes (HO-1 and $\mathrm{HO}-2$ ), while a greater quantity of $\mathrm{CO}$ is produced during stress conditions upon the induction of inducible HO-1. In view of the data presented here, we can speculate that to mimic an effective anti-aggregatory action of $\mathrm{CO}$ on platelets, slow but sustained kinetics of $\mathrm{CO}$ release is preferable as indicated by the significantly more potent antiaggregatory effect obtained with CORM-A1.

It has been consistently shown that $\mathrm{SGC}$ is strongly activated by NO and to a lesser extent by CO (Friebe et al. 1998; Hoenicka et al. 1999; Stone and Marletta 1998). Here, we found that in contrast to NO donors, the anti-aggregatory activity of CORM-A1 was not mediated by SGC. Indeed, in our experiments, ODQ, a potent and selective inhibitor of sGC (Garthwaite et al. 1995), completely reversed the anti-platelet effect of DEA-NO, while it was ineffective against CORM-A1. Furthermore, sildenafil, a phosphodiesterase (PDE)-5 inhibitor, failed to potentiate the effect of CO liberated by CORM-A1 in human platelets. At the same time, sildenafil amplified the antiaggregatory effect of NO released by DEA-NO. Previously, we demonstrated that CORM-3 inhibited platelets by a sGCindependent mechanism, while others had shown that high concentrations of gaseous CO (100\%) inhibited aggregation via activation of sGC (Brune et al. 1990). Here, using ODQ, a selective inhibitor of sGC, and using sildenafil, a PDE-5 inhibitor that inhibits degradation of cGMP, we provide evidence that inhibition of platelet aggregation by physiologically relevant concentrations of CO liberated by CORM-A1 was mediated by a sGC-independent mechanism. Apparently, in platelets as well as other tissues, $\mathrm{sGC}$ does not appear to be a primary target for the bioactivity of micromolar concentration of CO. Apart from sGC (Christodoulides et al. 1995; Furchgott and Jothianandan 1991; Hussain et al. 1997), there are a number of other possible targets for $\mathrm{CO}$ such as calcium-activated potassium channels (Wang and $\mathrm{Wu}$ 1997), cytochrome $\mathrm{P} 450$ (Coceani et al. 1997), mitochondrial respiratory chain (Lo
Iacono et al. 2011), or p38 MAPK (Amersi et al. 2002; Zhang et al. 2003). The role of each of these potential targets responsive to $\mathrm{CO}$ in mediating the anti-aggregatory effects of CO-RMs remains to be fully investigated.

Despite the fact that the specific target(s) for the antiaggregatory action of low concentrations of $\mathrm{CO}$ in platelets remain(s) unknown, our data point out that the use of CO-RM may represent a novel approach for selective and effective inhibition of platelets in vivo. We previously demonstrated that in contrast to $\mathrm{NO}$ and $\mathrm{PGI}_{2}, \mathrm{CO}$ effectively inhibited platelet aggregation even when platelets were excessively activated (Chlopicki et al. 2006). These results suggest that CO may play a role of a retaliatory mediator that comes into play when $\mathrm{NO}$ and $\mathrm{PGI}_{2}$ are insufficient to overcome excessive platelet activation, and this can be mimicked by CO-releasing agents.

The comparison between the effect of CO-RMs and NO donors on inhibition of platelet aggregation offers some additional points of discussion that should be taken into consideration. First of all, it is clear that all NO donors tested are more potent inhibitors of platelet aggregation than either CORM-3 or CORM-A1. Secondly, the slow CO releaser (CORM-A1, $t_{1 / 2}=21 \mathrm{~min}$ ) is in average 15 times more potent than the fast CO carrier (CORM-3, $t_{1 / 2}<1 \mathrm{~min}$ ) in inhibiting aggregation, and this is true both in PRP and WP. In the whole blood, CORM-A1 is still more potent than CORM-3 by approximately twofold. Interestingly, an opposite profile is observed for the NO donors since DETA-NO, which has a half-life of approximately $25 \mathrm{~min}$, is 150 times less potent than the fast NO donor DEA-NO $\left(t_{1 / 2}=1 \mathrm{~min}\right)$. Notably, the intriguing observation of our experiments is that the anti-aggregation effect of both NO donors and CO-RMs is ten times more pronounced in washed platelets than in PRP. Although the reduced effect on platelet aggregation in plasma may be predicted in the case of $\mathrm{NO}$ donors, this is not so intuitive and is rather unexpected for CO-releasing compounds. In fact, it is well established that $\mathrm{NO}$, apart from interacting strongly with ferrous $\left(\mathrm{Fe}^{2+}\right)$ and ferric $\left(\mathrm{Fe}^{3+}\right)$ metal centers, has a preferential reactivity with thiols and cysteine moieties present in proteins (Stamler et al. 2001; Stamler et al. 1992). Although this post-translational modification is illustrated in human red blood cells by S-nitrosylation of hemoglobin which has profound physiological implications on oxygen transport and delivery (Jia et al. 1996), the interaction of NO with cysteines in plasma is best exemplified by the rapid formation of S-nitrosoalbumin and possibly other stable circulating S-nitrosoproteins (Stamler et al. 2001; Stamler et al. 1992). The fact that in order to have the same degree of inhibition of aggregation, a higher concentration of $\mathrm{NO}$ donors is required in platelets present in plasma (PRP) compared to washed platelets which suggests that NO liberated from these agents is partially scavenged by the plasma components, possibly proteins rich in cysteines. 
In the case of $\mathrm{CO}$, it is more difficult to explain why CORMs elicit a reduced inhibition of platelet aggregation in plasma compared to washed platelets since only the presence of proteins containing ferrous iron $\left(\mathrm{Fe}^{2+}\right)$ or other transition metals with a specific redox state could act as effective scavengers of $\mathrm{CO}$. From the analysis of our results on the differential effects of CORM-3 and CORM-A1 in PRP, washed platelets, and in whole blood, it appears that the kinetics of $\mathrm{CO}$ release is an important factor in determining the potency of CO-RMs as anti-aggregatory agents. Our data using a sensitive $\mathrm{CO}$ electrode also reveal that indeed the total amount of CO liberated from CORM-A1 in PRP is much higher than the amount released from CORM-3; however, both the amount and the rate of CO release from CORM-A1 are markedly reduced in plasma compared to PBS especially when very high concentrations of CORM-A1 are used ( $3 \mathrm{mM})$. These data do not exclude the possibility that transition metal-containing proteins or enzymes present in plasma could act as potential scavengers of CO thus diminishing the potency of CORM-A1 in PRP. The general perception is that once present in the blood stream, CO would bind irreversibly to hemoglobin and eliminated through the lung by respiration, but a revision of this concept may be required as plasma components (i.e,. metal-containing proteins) may act either as potential targets of $\mathrm{CO}$ or carriers of $\mathrm{CO}$ to be transported either in circulating cells or tissues (Boczkowski et al. 2006; Foresti and Motterlini 2010). On the other hand, in the case of CORM3 , the increase in $\mathrm{COHb}$ is not substantial if one considers that heme concentration in human blood hemoglobin is in the order of $10 \mathrm{mM}$, and thus, $1 \mathrm{mM}$ CORM-3 should potentially generate $10 \% \mathrm{COHb}$. The fact that much less is detected with $1 \mathrm{mM}$ CORM-3 indicates that the total transfer of $\mathrm{CO}$ from the metal carbonyl to the heme in hemoglobin is not occurring or is somehow prevented by other factors. In the case of CORM-A1, the lower formation of $\mathrm{COHb}$ suggests that micromolar concentrations of CORMA1 may achieve effective anti-platelet effect without a significant $\mathrm{COHb}$ formation.

In conclusion, CORM-A1 that releases $\mathrm{CO}$ at a slower rate is a superior inhibitor of platelet aggregation than the fast $\mathrm{CO}$ carriers such as CORM-3. Furthermore, our results underscore the importance of both the chemical features of compounds releasing gaseous molecules and the kinetics of the gaseous mediator release that determine their anti-platelet efficacy. There is a clear difference between NO donors and CO-releasing molecules as regard to their mechanisms and pharmacodynamic characteristics of anti-platelet action that might help to determine an intracellular target for antiplatelet activity of low micromolar concentration of $\mathrm{CO}$ that is not shared by NO. Finally, our work points out that CORM-A1 displays promising anti-aggregatory activities, and thus, this compound or similar slow-releasers of $\mathrm{CO}$ should be exploited therapeutically further as an anti-thrombotic drug in vivo.

Acknowledgments We thank Prof. Brian Mann (University of Sheffield) for synthesizing CORM-3. Dr. Roberto Motterlini was recipient of a Visiting Professorship (2009-2011) from the Faculty of Medicine, University Paris Est, Creteil, France. This work was supported by the European Union from the resources of the European Regional Development Fund under the Innovative Economy Programme (grant coordinated by JCET-UJ, No. POIG.01.01.02-00-069/09). Supplementary funding was provided by the Polish Ministry of Science and Higher Education (N N405 260437).

Open Access This article is distributed under the terms of the Creative Commons Attribution License which permits any use, distribution, and reproduction in any medium, provided the original author(s) and the source are credited.

\section{References}

Amersi F, Shen XD, Anselmo D, Melinek J, Iyer S, Southard DJ, Katori M, Volk HD, Busuttil RW, Buelow R, Kupiec-Weglinski JW (2002) Ex vivo exposure to carbon monoxide prevents hepatic ischemia/reperfusion injury through p38 MAP kinase pathway. Hepatology 35:815-823

Boczkowski J, Poderoso JJ, Motterlini R (2006) CO-metal interaction: vital signaling from a lethal gas. Trends Biochem Sci 31:614-621

Born GV (1967) Possible mechanisms of platelet aggregation by ADP and of its inhibition. Thromb Diath Haemorrh Suppl 26:173-174

Brune B, Schmidt KU, Ullrich V (1990) Activation of soluble guanylate cyclase by carbon monoxide and inhibition by superoxide anion. Eur J Biochem 192:683-688

Cardinal DC, Flower RJ (1980) The electronic aggregometer: a novel device for assessing platelet behavior in blood. J Pharmacol Methods 3:135-158

Chlopicki S, Olszanecki R, Janiszewski M, Laurindo FR, Panz T, Miedzobrodzki J (2004) Functional role of NADPH oxidase in activation of platelets. Antioxid Redox Signal 6:691-698

Chlopicki S, Olszanecki R, Marcinkiewicz E, Lomnicka M, Motterlini R (2006) Carbon monoxide released by CORM-3 inhibits human platelets by a mechanism independent of soluble guanylate cyclase. Cardiovasc Res 71:393-401

Christodoulides N, Durante W, Kroll MH, Schafer AI (1995) Vascular smooth muscle cell heme oxygenases generate guanylyl cyclasestimulatory carbon monoxide. Circulation 91:2306-2309

Clark JE, Naughton P, Shurey S, Green CJ, Johnson TR, Mann BE, Foresti R, Motterlini R (2003) Cardioprotective actions by a water-soluble carbon monoxide-releasing molecule. Circ Res 93: e2-e8

Coceani F, Kelsey L, Seidlitz E, Marks GS, McLaughlin BE, Vreman HJ, Stevenson DK, Rabinovitch M, Ackerley C (1997) Carbon monoxide formation in the ductus arteriosus in the lamb: implications for the regulation of muscle tone. Br J Pharmacol 120:599608

Foresti R, Motterlini R (1999) The heme oxygenase pathway and its interaction with nitric oxide in the control of cellular homeostasis. Free Radic Res 31:459-475

Foresti R, Motterlini R (2010) Interaction of carbon monoxide with transition metals: evolutionary insights into drug target discovery. Curr Drug Targets 11:1595-1604

Foresti R, Hoque M, Bains S, Green CJ, Motterlini R (2003) Haem and nitric oxide: synergism in the modulation of the endothelial haem oxygenase-1 pathway. Biochem J 372:381-390 
Foresti R, Hammad J, Clark JE, Johnson TR, Mann BE, Friebe A, Green CJ, Motterlini R (2004) Vasoactive properties of CORM-3, a novel water-soluble carbon monoxide-releasing molecule. $\mathrm{Br} \mathrm{J}$ Pharmacol 142:453-460

Friebe A, Mullershausen F, Smolenski A, Walter U, Schultz G, Koesling D (1998) YC-1 potentiates nitric oxide- and carbon monoxideinduced cyclic GMP effects in human platelets. Mol Pharmacol 54:962-967

Furchgott RF, Jothianandan D (1991) Endothelium-dependent and -independent vasodilation involving cyclic GMP: relaxation induced by nitric oxide, carbon monoxide and light. Blood Vessels 28:52-61

Garthwaite J, Southam E, Boulton CL, Nielsen EB, Schmidt K, Mayer B (1995) Potent and selective inhibition of nitric oxide-sensitive guanylyl cyclase by $1 \mathrm{H}-[1,2,4]$ oxadiazolo[4,3-a]quinoxalin-1-one. Mol Pharmacol 48:184-188

Guo Y, Stein AB, Wu WJ, Tan W, Zhu X, Li QH, Dawn B, Motterlini R, Bolli R (2004) Administration of a CO-releasing molecule at the time of reperfusion reduces infarct size in vivo. Am J Physiol Heart Circ Physiol 286:H1649-H1653

Hoenicka M, Becker EM, Apeler H, Sirichoke T, Schroder H, Gerzer R, Stasch JP (1999) Purified soluble guanylyl cyclase expressed in a baculovirus/Sf9 system: stimulation by $\mathrm{YC}-1$, nitric oxide, and carbon monoxide. J Mol Med 77:14-23

Hussain AS, Marks GS, Brien JF, Nakatsu K (1997) The soluble guanylyl cyclase inhibitor $1 \mathrm{H}-[1,2,4]$ oxadiazolo[4,3-alpha]quinoxalin1-one (ODQ) inhibits relaxation of rabbit aortic rings induced by carbon monoxide, nitric oxide, and glyceryl trinitrate. Can J Physiol Pharmacol 75:1034-1037

Lo Iacono L, Boczkowski J, Zini R, Salouage I, Berdeaux A, Motterlini R, Morin D (2011) A carbon monoxide-releasing molecule (CORM3) uncouples mitochondrial respiration and modulates the production of reactive oxygen species. Free Radic Biol Med 50:1556-1564

Jia L, Bonaventura C, Bonaventura J, Stamler JS (1996) Snitrosohaemoglobin: a dynamic activity of blood involved in vascular control. Nature 380:221-226

Johnson TR, Mann BE, Clark JE, Foresti R, Green CJ, Motterlini R (2003) Metal carbonyls: a new class of pharmaceuticals? Angew Chem Int Ed Engl 42:3722-3729

Kalinowski L, Dobrucki LW, Szczepanska-Konkel M, Jankowski M, Martyniec L, Angielski S, Malinski T (2003) Third-generation beta-blockers stimulate nitric oxide release from endothelial cells through ATP efflux: a novel mechanism for antihypertensive action. Circulation 107:2747-2752

Maines MD, Kappas A (1977) Enzymatic oxidation of cobalt protoporphyrin IX: observations on the mechanism of heme oxygenase action. Biochemistry 16:419-423

Motterlini R (2007) Carbon monoxide-releasing molecules (CO-RMs): vasodilatory, anti-ischaemic and anti-inflammatory activities. Biochem Soc Trans 35:1142-1146
Motterlini R, Otterbein LE (2010) The therapeutic potential of carbon monoxide. Nat Rev Drug Discov 9:728-743

Motterlini R, Clark JE, Foresti R, Sarathchandra P, Mann BE, Green CJ (2002) Carbon monoxide-releasing molecules: characterization of biochemical and vascular activities. Circ Res 90:E17-E24

Motterlini R, Mann BE, Johnson TR, Clark JE, Foresti R, Green CJ (2003) Bioactivity and pharmacological actions of carbon monoxide-releasing molecules. Curr Pharm Des 9:2525-2539

Motterlini R, Sawle P, Hammad J, Bains S, Alberto R, Foresti R, Green CJ (2005) CORM-A1: a new pharmacologically active carbon monoxide-releasing molecule. FASEB J 19:284-286

Otterbein LE, Bach FH, Alam J, Soares M, Tao LH, Wysk M, Davis RJ, Flavell RA, Choi AM (2000) Carbon monoxide has antiinflammatory effects involving the mitogen-activated protein kinase pathway. Nat Med 6:422-428

Otterbein LE, Zuckerbraun BS, Haga M, Liu F, Song R, Usheva A, Stachulak C, Bodyak N, Smith RN, Csizmadia E, Tyagi S, Akamatsu Y, Flavell RJ, Billiar TR, Tzeng E, Bach FH, Choi AM, Soares MP (2003) Carbon monoxide suppresses arteriosclerotic lesions associated with chronic graft rejection and with balloon injury. Nat Med 9:183-190

Radomski MW, Palmer RM, Read NG, Moncada S (1988) Isolation and washing of human platelets with nitric oxide. Thromb Res 50:537-546

Sawle P, Foresti R, Mann BE, Johnson TR, Green CJ, Motterlini R (2005) Carbon monoxide-releasing molecules (CO-RMs) attenuate the inflammatory response elicited by lipopolysaccharide in RAW264.7 murine macrophages. Br J Pharmacol 145:800-810

Stamler JS, Simon DI, Osborne JA, Mullins ME, Jaraki O, Michel T, Singel DJ, Loscalzo J (1992) S-nitrosylation of proteins with nitric oxide: synthesis and characterization of biologically active compounds. Proc Natl Acad Sci USA 89:444-448

Stamler JS, Lamas S, Fang FC (2001) Nitrosylation: the prototypic redox-based signaling mechanism. Cell 106:675-683

Stone JR, Marletta MA (1998) Synergistic activation of soluble guanylate cyclase by YC-1 and carbon monoxide: implications for the role of cleavage of the iron-histidine bond during activation by nitric oxide. Chem Biol 5:255-261

Tenhunen R, Marver HS, Schmid R (1969) The enzymatic conversion of hemoglobin to bilirubin. Trans Assoc Am Physicians 82:363-371

Utz J, Ullrich V (1991) Carbon monoxide relaxes ileal smooth muscle through activation of guanylate cyclase. Biochem Pharmacol 41:1195-1201

Wang R, Wu L (1997) The chemical modification of KCa channels by carbon monoxide in vascular smooth muscle cells. J Biol Chem 272:8222-8226

Zhang X, Shan P, Otterbein LE, Alam J, Flavell RA, Davis RJ, Choi AM, Lee PJ (2003) Carbon monoxide inhibition of apoptosis during ischemia-reperfusion lung injury is dependent on the p38 mitogen-activated protein kinase pathway and involves caspase 3 . J Biol Chem 278:1248-1258 